CONFORMAL GEOMETRY AND DYNAMICS

An Electronic Journal of the American Mathematical Society

Volume 1, Pages 87-103 (December 9, 1997)

S 1088-4173(97)00020-9

\title{
GEOMETRIC INTERSECTION NUMBERS ON A FOUR-PUNCTURED SPHERE
}

\author{
YUNGYEN CHIANG
}

\begin{abstract}
Let $\mathcal{G}_{4}$ be the space of all simple closed geodesics on the punctured sphere $\Sigma_{4}$. We construct an explicit homeomorphism of the completion of $\mathcal{G}_{4}$ onto a circle by using geometric intersection numbers. Also, we relate these geometric intersection numbers to trace polynomials of transformations corresponding to geodesics in $\mathcal{G}_{4}$ in a representation of $\pi_{1}\left(\Sigma_{4}\right)$ into $\operatorname{PSL}(2, \mathbf{C})$.
\end{abstract}

\section{INTRODUCTION}

Thurston proved that the simple geodesics on a Riemann surface can be made into a topological space and that this space is homeomorphic to a sphere whose dimension depends on the topology of the surface. We shall be interested in the space of simple geodesics on a 4-punctured sphere. By Thurston's result, this space is homeomorphic to a circle. The aim of this article is to give an explicit construction of this homeomorphism.

The motivation for this work was an attempt to generalize the pleating coordinates defined for the Maskit embedding of the Teichmüller space of a punctured torus ([4]) to four-times punctured spheres ([2]). The construction given here is used to relate the asymptotic behavior of the trace of an element of a marked Kleinian group representing a simple closed geodesic to its intersections with the marking curves.

We present the construction and show the traces here because it is of interest in its own right.

The rest of the paper is organized as follows. In $\S 1$, we recall some basic definitions and set up some notations. A regular $B$-group $G$ will be given in this section to represent a 4-punctured sphere $\Sigma_{4}$ and two thrice-punctured spheres.

In $\S 2$, the set $\mathcal{G}_{4}$ of all simple closed geodesics is described by using a technique developed in [1]. Each $\gamma \in \mathcal{G}_{4}$ is associated with two integers $I_{X}(\gamma)$ and $N(\gamma)$ such that $I_{X}(\gamma)$ and $|N(\gamma)|$ are geometric intersection numbers of $\gamma \in \mathcal{G}_{4}$ with two specific geodesics in $\mathcal{G}_{4}$. By using these two integers, a formula for the geometric intersection number of any two geodesics $\gamma$ and $\gamma^{\prime}$ in $\mathcal{G}_{4}$ is given in Theorem 2.6. In Theorem 2.5, we give a homeomorphism $\Psi$ of the space of simple geodesics on $\Sigma_{4}$ onto $\mathbb{R} \cup\{\infty\}$ with $\Psi(\gamma)=\frac{N(\gamma)}{I_{X}(\gamma)}$ for all $\gamma \in \mathcal{G}_{4}$.

In the last section, for each simple closed geodesic $\gamma$ on $\Sigma_{4}$, we find a word $W(\gamma)$ in $G$ to represent it (Theorem 3.2). Then, we consider the deformation space of $G$, which is a holomorphic family of regular $B$-groups $G_{\mu}$ quasiconformally conjugate

Received by the editors April 28, 1997 and, in revised form, September 6, 1997.

1991 Mathematics Subject Classification. Primary 30Fxx; Secondary 57-XX.

(C)1997 American Mathematical Society 
to $G$, where $\mu$ are complex numbers. For every $\mu$ and for every $\gamma$, there is a word $W(\gamma ; \mu) \in G_{\mu}$ representing $\gamma$. The trace of $W(\gamma ; \mu)$ is a polynomial in $\mu$. We compute higher order terms of the trace of $W(\gamma ; \mu)$ in Theorem 3.4. The degree and coefficients of higher order terms of the trace polynomial are written in terms of $I_{X}(\gamma)$ and $N(\gamma)$.

\section{ACKNOWLEDGEMENT}

The author would like to thank Professor L. Keen for her encouragement and for her comments.

\section{Basic Definitions}

Let $\Sigma_{4}$ be a 4 -punctured sphere equipped with a hyperbolic metric. A simple closed curve on $\Sigma_{4}$ is called essential if it is neither homotopically trivial nor homotopically equivalent to a puncture of $\Sigma_{4}$. Let $\mathcal{G}_{4}$ be the set of all free homotopy classes of non-oriented essential simple closed curves on $\Sigma_{4}$. Every element in $\mathcal{G}_{4}$ contains a unique geodesic $\gamma$ on $\Sigma_{4}$. By abuse of notation, we shall also use $\gamma$ for the free homotopy class containing $\gamma$.

We provide $\mathcal{G}_{4}$ with the discrete topology. Let $\left[\mathcal{G}_{4}, \mathbb{R}_{+}\right]$be the set of all functions from $\mathcal{G}_{4}$ into the set $\mathbb{R}_{+}$of all non-negative real numbers. $\left[\mathcal{G}_{4}, \mathbb{R}_{+}\right]$is provided with the compact-open topology. It is well known that $\left[\mathcal{G}_{4}, \mathbb{R}_{+}\right]$is homeomorphic to the product space $\prod_{\gamma \in \mathcal{G}_{4}} \mathbb{R}_{+}^{\gamma}$, where each $\mathbb{R}_{+}^{\gamma}$ is a copy of $\mathbb{R}_{+}$. Two elements $f$ and $g$ of $\left[\mathcal{G}_{4}, \mathbb{R}_{+}\right]-\{0\}$ are called projectively equivalent if there is a positive number $t$ such that $f=t g$. Let $\mathrm{P}\left[\mathcal{G}_{4}, \mathbb{R}_{+}\right]$be the set of all projective equivalence classes in $\left[\mathcal{G}_{4}, \mathbb{R}_{+}\right]-\{0\}$ provided with the quotient topology. Let

$$
\pi:\left[\mathcal{G}_{4}, \mathbb{R}_{+}\right]-\{0\} \longrightarrow \mathrm{P}\left[\mathcal{G}_{4}, \mathbb{R}_{+}\right]
$$

be the quotient map. One can show that $\pi$ is a closed and open map.

For any two elements $\gamma$ and $\gamma^{\prime}$ of $\mathcal{G}_{4}$, we denote by $i\left(\gamma, \gamma^{\prime}\right)$ the geometric intersection number of $\gamma$ with $\gamma^{\prime}$. Each $\gamma \in \mathcal{G}_{4}$ defines a function $\mathrm{I}_{\gamma}: \mathcal{G}_{4} \longrightarrow \mathbb{R}_{+}$given by

$$
\mathrm{I}_{\gamma}(\alpha)=i(\gamma, \alpha) \quad \text { for } \alpha \in \mathcal{G}_{4} .
$$

Let $\mathcal{I}: \mathcal{G}_{4} \longrightarrow\left[\mathcal{G}_{4}, \mathbb{R}_{+}\right]$be defined by

$$
\mathcal{I}(\gamma)=\mathrm{I}_{\gamma} \quad \text { for } \gamma \in \mathcal{G}_{4}
$$

It is a well known fact that the composition $\pi \mathcal{I}$ is injective (see [3]). This allows us to identify $\mathcal{G}_{4}$ with $\pi \mathcal{I}\left(\mathcal{G}_{4}\right)$. Thurston proved that the closure $\overline{\pi \mathcal{I}\left(\mathcal{G}_{4}\right)}$ of $\pi \mathcal{I}\left(\mathcal{G}_{4}\right)$ in $\mathrm{P}\left[\mathcal{G}_{4}, \mathbb{R}_{+}\right]$is homeomorphic to a circle. In $\S 2$, we shall construct a homeomorphism of $\overline{\pi \mathcal{I}\left(\mathcal{G}_{4}\right)}$ onto a circle.

Note that an element $\mathcal{L}$ of $\mathrm{P}\left[\mathcal{G}_{4}, \mathbb{R}_{+}\right]$is in $\overline{\pi \mathcal{I}\left(\mathcal{G}_{4}\right)}$ if and only if for any $\ell$ in $\left[\mathcal{G}_{4}, \mathbb{R}_{+}\right]-\{0\}$ with $\pi(\ell)=\mathcal{L}$ there is a sequence $\left\{t_{k}\right\}_{k=1}^{\infty}$ of positive numbers and a sequence $\left\{\gamma_{k}\right\}_{k=1}^{\infty}$ in $\mathcal{G}_{4}$ such that the sequence $\left\{t_{k} \mathrm{I}_{\gamma_{k}}\right\}_{k=1}^{\infty}$ converges to $\ell$. A sequence $\left\{\ell_{k}\right\}_{k=1}^{\infty}$ in $\left[\mathcal{G}_{4}, \mathbb{R}_{+}\right]$is called convergent to $\ell \in\left[\mathcal{G}_{4}, \mathbb{R}_{+}\right]$if for every $\gamma \in \mathcal{G}_{4}$ the sequence $\left\{\ell_{k}(\gamma)\right\}_{k=1}^{\infty}$ converges to $\ell(\gamma)$.

To enumerate free homotopy classes in $\mathcal{G}_{4}$, we use a Kleinian group $G$ whose regular set has an invariant connected component $\Omega_{0}$ such that $\Omega_{0} / G=\Sigma_{4}$. Since this 

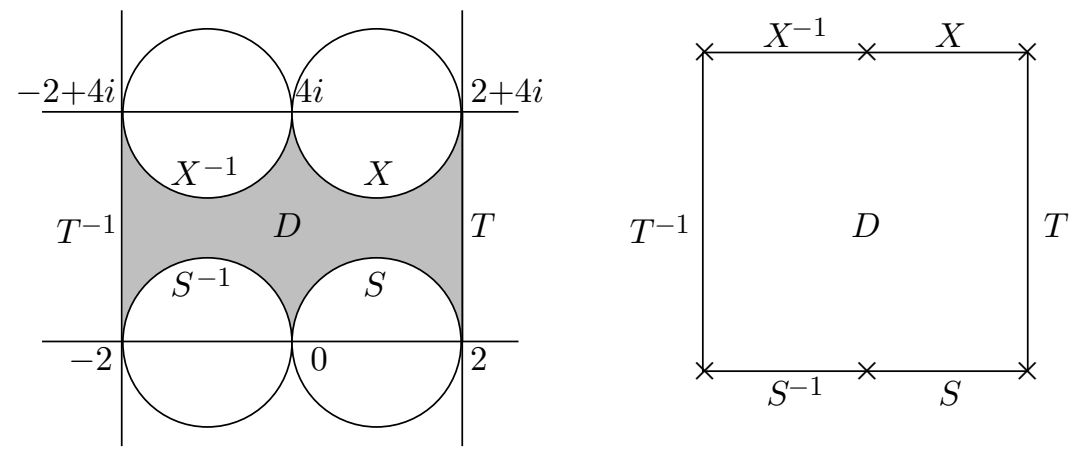

Figure 1. The fundamental domain $D$

is a topological problem, we may choose a $B$-group $G$ representing a 4 -punctured sphere and two thrice-punctured spheres. Let

$$
S=\left(\begin{array}{ll}
1 & 0 \\
1 & 1
\end{array}\right), \quad T=\left(\begin{array}{ll}
1 & 4 \\
0 & 1
\end{array}\right), \quad \text { and } \quad X=\left(\begin{array}{cc}
1+4 i & 16 \\
1 & 1-4 i
\end{array}\right) .
$$

By using Maskit's first combination theorem ([6], Theorem VII.C.2.), one can prove that the subgroup $G$ of $P S L(2, \mathbf{C})$ generated by $S, T$ and $X$ is a $B$-group representing a 4-punctured sphere and two thrice-punctured spheres. We choose a connected and simply connected fundamental domain $\mathcal{D}$ for $G$ acting on the invariant connected component of the regular set of $G$ (see left of Figure 1).

For simplicity, we shall schematically draw $\mathcal{D}$ as given in the right of Figure 1. If $s$ and $s^{\prime}$ are sides of $\mathcal{D}$, and $E \in\left\{S^{ \pm}, T^{ \pm}, X^{ \pm}\right\}$with $E(s)=s^{\prime}$, then we label $E^{-1}$ beside $s$, and label $E$ beside $s^{\prime}$. A side $s$ of $\mathcal{D}$ is called the $E$-side of $\mathcal{D}$ if $s$ is labelled by $E$. With this labelling, we can easily write words in $G$ to represent curves in $\mathcal{G}_{4}$.

It is easy to see that if $\gamma$ and $\gamma^{\prime}$ are two essential simple closed curves on $\Sigma_{4}$ with the same underlying set but opposite orientations, and if $W$ is a word in $G$ representing $\gamma$, then $\gamma^{\prime}$ is represented by $W^{-1}$. Because we are only interested in non-oriented essential simple closed curves, we shall not distinguish $W^{-1}$ from $W$. On the other hand, since every free homotopy class of a simple closed curve corresponds to a unique conjugacy class of an element of $G$, we shall not distinguish a word in $G$ from its conjugates. In particular, a cyclic permutation of a word $W$ in $G$ is conjugate to $W$ in $G$. We shall identify these words and call any one of them a cyclic word.

A word $W=E_{1} \cdots E_{m}$ in $G$ will be called a reduced word if for each $j$,

$$
E_{j} \in\left\{S^{ \pm}, T^{ \pm}, X^{ \pm}\right\} \text {, and } E_{j} E_{j+1} \neq \mathbf{1} \text {, for } j=1, \ldots, m-1,
$$

where 1 is the identity of $G$. When $W$ represents a curve in $\mathcal{G}_{4}$, we also require that $E_{m} E_{1} \neq 1$. For every $j=1, \ldots, m$, and every positive integer $k<m$, we call $E_{j+1} \cdots E_{j+k}$ a subword of $W$, where $E_{m+1}=E_{1}$.

Let $\Pi: \mathcal{D} \longrightarrow \Sigma_{4}$ be the canonical projection. For $E \in\left\{S^{ \pm}, T^{ \pm}, X^{ \pm}\right\}$, let $\gamma_{E}$ denote the image of the $E$-side under $\Pi$. $\gamma_{E}$ is a simple $\operatorname{arc}$ on $\Sigma_{4}$ joining two punctures of $\Sigma_{4}$.

For every $\gamma \in \mathcal{G}_{4}, \Pi^{-1}(\gamma)$ is a disjoint union of a finite number of simple arcs in $\mathcal{D}$ with endpoints on the sides of $\mathcal{D}$. We call each of these simple arcs a strand of 
$\gamma . \gamma$ has exactly $k \geq 0$ strands with endpoints on the $E$-side if and only if it has exactly $k \geq 0$ strands with endpoints on the $E^{-1}$-side. We denote by $I_{E}(\gamma)$ the geometric intersection number of $\gamma$ with $\gamma_{E}$. Then $I_{E}(\gamma)$ is the number of points which are endpoints of strands of $\gamma$ on the $E$-side (or the $E^{-1}$-side).

\section{The Space $\mathcal{G}_{4}$}

In this section, we shall construct a homeomorphism $\Psi$ of $\overline{\pi \mathcal{I}\left(\mathcal{G}_{4}\right)}$ onto $\hat{\mathbb{R}}=$ $\mathbb{R} \cup\{\infty\}$ so that $\Psi\left(\mathcal{G}_{4}\right)=\mathbf{Q} \cup\{\infty\}$, where $\mathbb{R}$ is the set of all real numbers, $\hat{\mathbb{R}}$ is the one-point compactification of $\mathbb{R}$, and $\mathbf{Q} \subset \mathbb{R}$ is the set of all rational numbers. To construct the homeomorphism $\Psi$, we shall first enumerate the space $\mathcal{G}_{4}$ by using a technique developed by Birman and Series [1].

An enumeration of $\mathcal{G}_{4}$ is given by Keen and Series in [5] as follows. Let $L=$ $\{m+n i: m, n$ are integers $\}$, and let $\Gamma$ be the group of Möbius transformations generated by the transformations

$$
z \longmapsto z+2, \quad z \longmapsto z+2 i \text { and } z \longmapsto-z .
$$

The quotient $(\mathbf{C}-L) / \Gamma$ is a four-punctured sphere. For each $m \in \mathbf{Q} \cup\{\infty\}$, let $\ell(m)$ be a straight line in $\mathbf{C}-L$ of slope $m$. Keen and Series enumerate the space $\mathcal{G}_{4}$ by identifying each free homotopy class $\gamma \in \mathcal{G}_{4}$ with a number $m \in \mathbf{Q} \cup\{\infty\}$ whenever $\gamma$ is represented by the projection of $\ell(m)$ on $(\mathbf{C}-L) / \Gamma$.

The enumeration of $\mathcal{G}_{4}$ that will be given in this section is essentially the same as that given in [5]. Our enumeration can be read off directly from some numbers of strands of geodesics in $\mathcal{G}_{4}$, and these numbers relate directly to trace polynomials of transformations in $G$ representing geodesics in $\mathcal{G}_{4}$ (see Theorem 3.4).

Let $\mathcal{S}\left(\Sigma_{4}\right)$ be the set of all isotopy classes of families of finite unions of pairwise disjoint simple loops on $\Sigma_{4}$, subject to the restriction that if $\Lambda$ is in $\mathcal{S}\left(\Sigma_{4}\right)$, then no connected component of $\Lambda$ is either homotopically trivial or homotopically equivalent to a puncture of $\Sigma_{4}$. It is clear that $\mathcal{G}_{4}$ is a subset of $\mathcal{S}\left(\Sigma_{4}\right)$. In fact, each $\Lambda \in \mathcal{S}\left(\Sigma_{4}\right)$ is a finite number of copies of a geodesic $\gamma$ in $\mathcal{G}_{4}$; more precisely, each connected component of $\Lambda$ is homotopically equivalent to $\gamma$.

Let $\Pi: \mathcal{D} \longrightarrow \Sigma_{4}$ be the canonical projection. For each $\Lambda \in \mathcal{S}\left(\Sigma_{4}\right), \Pi^{-1}(\Lambda)$ is a union of a finite number of simple arcs in $\mathcal{D}$. Each of these simple arcs is called a strand of $\Lambda$. For $E_{1}$ and $E_{2}$ in $\left\{S^{ \pm}, T^{ \pm}, X^{ \pm}\right\}$, let $n_{\Lambda}\left(E_{1}, E_{2}\right)$ be the number of strands of $\Lambda$ which connect the $E_{1}$-side to the $E_{2}$-side of $\mathcal{D}$. Now, for each $E \in\left\{S^{ \pm}, T^{ \pm}, X^{ \pm}\right\}$, we choose a point $P(E)$ on the $E$-side of $\mathcal{D}$ so that $P\left(E^{-1}\right)$ and $P(E)$ are identified by $E$. Let $\tau(\Lambda)$ be the weighted graph obtained by collapsing all those strands of $\Lambda$ which join a given pair of sides $E_{1}$ and $E_{2}$ of $\mathcal{D}$ to a single arc from $P\left(E_{1}\right)$ to $P\left(E_{2}\right)$, labelled by the number $n_{\Lambda}\left(E_{1}, E_{2}\right)$ of strands which were collapsed. Birman and Series call the weighted graph $\tau(\Lambda)$ a $\pi_{1}$-train track. For convenience, we set $n_{\Lambda}\left(E_{1}, E_{2}\right)=0$ if there is no arc in $\tau(\Lambda)$ joining $P\left(E_{1}\right)$ to $P\left(E_{2}\right)$.

Let $\Lambda_{1}, \Lambda_{2}$ and $\Lambda$ be any three elements of $\mathcal{S}\left(\Sigma_{4}\right)$. If, as sets of points of $\mathcal{D}, \tau(\Lambda)$ is the union of $\tau\left(\Lambda_{1}\right)$ and $\tau\left(\Lambda_{2}\right)$, and if there are two fixed non-negative integers $a$ and $b$ with $a^{2}+b^{2} \neq 0$ satisfying

$$
n_{\Lambda}\left(E_{1}, E_{2}\right)=a n_{\Lambda_{1}}\left(E_{1}, E_{2}\right)+b n_{\Lambda_{2}}\left(E_{1}, E_{2}\right)
$$

for any pair of sides $E_{1}, E_{2}$ of $\mathcal{D}$, then we shall write

$$
\Lambda=a \Lambda_{1}+b \Lambda_{2} \text {. }
$$




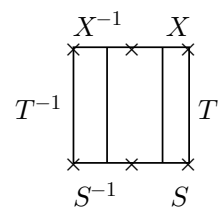

(1)

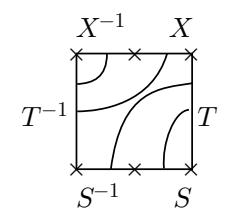

$(4)$

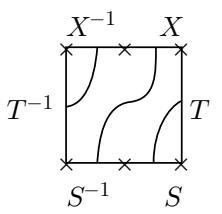

(2)

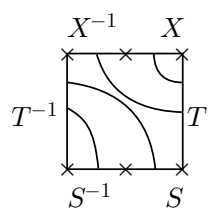

$(5)$

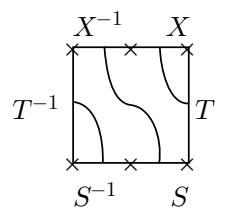

(3)

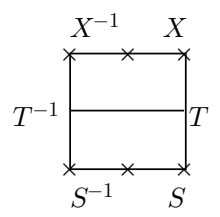

(6)

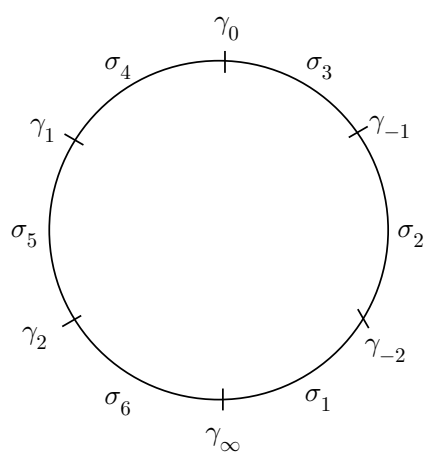

Figure 2. (1) $\gamma_{0}, \quad$ (2) $\gamma_{1}, \quad$ (3) $\gamma_{-1}, \quad$ (4) $\gamma_{2}, \quad$ (5) $\gamma_{-2}, \quad$ (6) $\gamma_{\infty}$

For $x \in\{0, \pm 1, \pm 2, \infty\}$, let $\gamma_{x}$ be the simple closed geodesic in $\mathcal{G}_{4}$ represented by $W_{x} \in G$ given as the following:

$$
\begin{gathered}
W_{0}=X^{-1} S, \quad W_{1}=X T^{-1} S, \quad W_{2}=T X T^{-1} S^{-1}, \\
W_{-1}=X^{-1} T S^{-1}, \quad W_{-2}=T^{-1} X^{-1} T S, \quad W_{\infty}=T ;
\end{gathered}
$$

see Figure 2. It is easy to see that every $\Lambda \in \mathcal{S}\left(\Sigma_{4}\right)$ can be written as one of the following:

$$
a \gamma_{0}+b \gamma_{1}, \quad a \gamma_{1}+b \gamma_{2}, \quad a \gamma_{2}+b \gamma_{\infty}, \quad a \gamma_{0}+b \gamma_{-1}, \quad a \gamma_{-1}+b \gamma_{-2}, \quad a \gamma_{-2}+b \gamma_{\infty},
$$

where $a, b \geq 0$ are integers with $a^{2}+b^{2} \neq 0$. Moreover, $\Lambda \in \mathcal{G}_{4}$ if and only if $a$ and $b$ are relatively prime. Let

$$
\begin{aligned}
& \sigma_{1}=\left\{a \gamma_{-2}+b \gamma_{\infty}: a, b \text { are relatively prime non-negative integers }\right\}, \\
& \sigma_{2}=\left\{a \gamma_{-1}+b \gamma_{-2}: a, b \text { are relatively prime non-negative integers }\right\}, \\
& \sigma_{3}=\left\{a \gamma_{0}+b \gamma_{-1}: a, b \text { are relatively prime non-negative integers }\right\}, \\
& \sigma_{4}=\left\{a \gamma_{0}+b \gamma_{1}: a, b \text { are relatively prime non-negative integers }\right\}, \\
& \sigma_{5}=\left\{a \gamma_{1}+b \gamma_{2}: a, b \text { are relatively prime non-negative integers }\right\}, \\
& \sigma_{6}=\left\{a \gamma_{2}+b \gamma_{\infty}: a, b \text { are relatively prime non-negative integers }\right\} .
\end{aligned}
$$

Let $\Theta, \mathcal{T}_{1}$ and $\mathcal{T}_{2}$ be the automorphisms of $G$ defined by

$$
\begin{aligned}
\Theta & : \quad S \longmapsto S^{-1}, \quad T \longmapsto T^{-1}, \quad X \longmapsto X^{-1}, \\
\mathcal{T}_{1} & : \quad S \longmapsto S^{-1} T, \quad T \longmapsto T, \quad X \longmapsto X, \\
\mathcal{T}_{2} & : \quad S \longmapsto S, \quad T \longmapsto S T^{-1} X \quad X \longmapsto X .
\end{aligned}
$$

$\Theta$ induces an (orientation reversing) homeomorphism of $\Sigma_{4}$ onto itself, and $\mathcal{T}_{j}$ induces an (orientation preserving) homeomorphism of $\Sigma_{4}$ onto itself, also denoted by $\Theta$ and $\mathcal{T}_{j}$, respectively. $\mathcal{T}_{1}$ interchanges the two punctures of $\Sigma_{4}$ corresponding to the fixed points of $S$ and $T S^{-1}$, and $\mathcal{T}_{2}$ interchanges the two punctures of $\Sigma_{4}$ corresponding to the fixed points of $T^{-1} S$ and $X^{-1} T$.

A result of Birman and Series [1] says that $\Theta, \mathcal{T}_{1}$ and $\mathcal{T}_{2}$ act on $\mathcal{S}\left(\Sigma_{4}\right)$ "linearly". More precisely, for $\varphi=\Theta$ or $\mathcal{T}_{j}$, if $\gamma, \gamma^{\prime} \in \sigma_{i}$ for some $i$, and if $\varphi(\gamma), \varphi\left(\gamma^{\prime}\right) \in \sigma_{j}$ for 
some $j$, then

$$
\varphi\left(a \gamma+b \gamma^{\prime}\right)=a \varphi(\gamma)+b \varphi\left(\gamma^{\prime}\right)
$$

where $a$ and $b$ are any two non-negative integers with $a^{2}+b^{2} \neq 0$.

Let $\hat{\mathcal{G}_{4}}=\mathcal{G}_{4}-\left\{\gamma_{\infty}\right\}$. We associate to each $\gamma \in \hat{\mathcal{G}_{4}}$ the integer $N(\gamma)$ defined as follows:

$$
\begin{aligned}
N(\gamma) \geq & 0 \text { when } \gamma \in \sigma_{4} \cup \sigma_{5} \cup \sigma_{6}, \\
N(\gamma) \leq & 0 \text { when } \gamma \in \sigma_{1} \cup \sigma_{2} \cup \sigma_{3}, \text { and } \\
|N(\gamma)|= & \#\left(\text { strands of } \gamma \text { joining the } T^{-1} \text {-side and the } T\right. \text {-side) } \\
& +\# \text { (strands of } \gamma \text { joining the } T^{\varepsilon} \text {-side and the } E^{\delta} \text {-side) } \\
= & I_{T}(\gamma),
\end{aligned}
$$

where $\varepsilon, \delta \in\{1,-1\}$ and $E \in\{X, S\}$. Set

$$
\begin{aligned}
& \mathcal{G}_{4}^{+}=\left\{\gamma \in \hat{\mathcal{G}}_{4}: N(\gamma) \geq 0\right\}=\sigma_{4} \cup \sigma_{5} \cup \sigma_{6}-\left\{\gamma_{\infty}\right\}, \\
& \mathcal{G}_{4}^{-}=\left\{\gamma \in \hat{\mathcal{G}_{4}}: N(\gamma) \leq 0\right\}=\sigma_{1} \cup \sigma_{2} \cup \sigma_{3}-\left\{\gamma_{\infty}\right\} .
\end{aligned}
$$

Lemma 2.1. Let $\gamma \in \hat{\mathcal{G}}_{4}$. Then there is an integer $k$ depending on $\gamma$ such that $\mathcal{T}_{1}^{k}(\gamma) \in \sigma_{4}$.

Proof. It is easy to see that this lemma holds for $\gamma \in \bigcup_{j=2}^{5} \sigma_{j}$. Next, we shall prove that this is true for $\gamma \in \sigma_{6}-\left\{\gamma_{\infty}, \gamma_{2}\right\}$. By a similar argument, the assertion will follow for $\gamma \in \sigma_{1}-\left\{\gamma_{\infty}, \gamma_{-2}\right\}$.

Write $\gamma=a \gamma_{2}+b \gamma_{\infty}$, where $a, b>0$ and $\operatorname{gcd}(a, b)=1$. There exist nonnegative integers $k$ and $c<a$ such that $b=a k+c$. Since $\mathcal{T}_{1}^{-1}\left(\gamma_{2}+\gamma_{\infty}\right)=\gamma_{2}$ and $\mathcal{T}_{1}\left(\gamma_{\infty}\right)=\gamma_{\infty}$, then

$$
\begin{aligned}
\mathcal{T}_{1}^{-k}(\gamma) & =a \gamma_{2}+c \gamma_{\infty}=(a-c) \gamma_{2}+c\left(\gamma_{2}+\gamma_{\infty}\right), \\
\mathcal{T}_{1}^{-k-2}(\gamma) & =\mathcal{T}_{1}^{-1}\left((a-c) \gamma_{1}+c \gamma_{2}\right)=(a-c) \gamma_{0}+c \gamma_{1} .
\end{aligned}
$$

Lemma 2.2. If $\gamma \in \hat{\mathcal{G}_{4}}$, then

(1) $I_{X}(\Theta(\gamma))=I_{X}(\gamma), N(\Theta(\gamma))=-N(\gamma)$, and

(2) $I_{X}\left(\mathcal{T}_{1}(\gamma)\right)=I_{X}(\gamma), N\left(\mathcal{T}_{1}(\gamma)\right)=N(\gamma)+I_{X}(\gamma)$.

Proof. (1) follows immediately from the definition of $N(\gamma)$ and that of $I_{X}(\gamma)$.

For the proof of (2), we first assume that $\gamma \in \mathcal{G}_{4}^{+}$.

Case 1. If $\gamma=a \gamma_{0}+b \gamma_{1}$, then $N(\gamma)=b$ and $I_{X}(\gamma)=a+b$.

$$
\begin{aligned}
\mathcal{T}_{1}(\gamma) & =a \gamma_{1}+b \gamma_{2}, \quad I_{X}\left(\mathcal{T}_{1}(\gamma)\right)=a+b=I_{X}(\gamma), \\
N\left(\mathcal{T}_{1}(\gamma)\right) & =a+2 b=N(\gamma)+I_{X}(\gamma) .
\end{aligned}
$$

Case 2. If $\gamma=a \gamma_{1}+b \gamma_{2}$, then $N(\gamma)=a+2 b$ and $I_{X}(\gamma)=a+b$.

$$
\begin{aligned}
\mathcal{T}_{1}(\gamma) & =(a+b) \gamma_{2}+b \gamma_{\infty}, \quad I_{X}\left(\mathcal{T}_{1}(\gamma)\right)=a+b=I_{X}(\gamma), \\
N\left(\mathcal{T}_{1}(\gamma)\right) & =2(a+b)+b=N(\gamma)+I_{X}(\gamma) .
\end{aligned}
$$

Case 3. If $\gamma=a \gamma_{2}+b \gamma_{\infty}$, then $N(\gamma)=2 a+b$ and $I_{X}(\gamma)=a$.

$$
\begin{aligned}
\mathcal{T}_{1}(\gamma) & =a \gamma_{2}+(a+b) \gamma_{\infty}, \quad I_{X}\left(\mathcal{T}_{1}(\gamma)\right)=a=I_{X}(\gamma) \\
N\left(\mathcal{T}_{1}(\gamma)\right) & =3 a+b=N(\gamma)+I_{X}(\gamma) .
\end{aligned}
$$




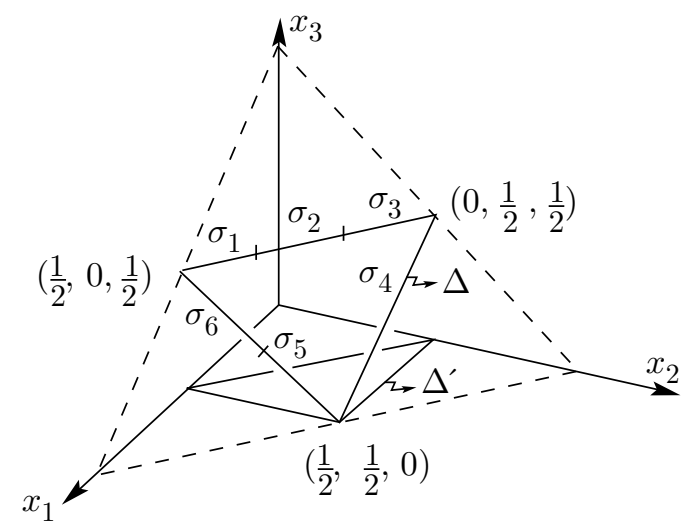

Figure 3 . The triangle $\Delta$ and the triangle $\Delta^{\prime}$

Now, let $\gamma \in \mathcal{G}_{4}^{-}$. Since $\Theta \mathcal{T}_{1}=\mathcal{T}_{1}^{-1} \Theta$, then

$$
\begin{gathered}
\mathcal{T}_{1}(\gamma)=\Theta \mathcal{T}_{1}^{-1} \Theta(\gamma), \quad I_{X}\left(\mathcal{T}_{1}(\gamma)\right)=I_{X}(\gamma), \quad \text { and } \\
N\left(\Theta \mathcal{T}_{1}^{-1} \Theta(\gamma)\right)=-N\left(\mathcal{T}_{1}^{-1} \Theta(\gamma)\right)=-N(\Theta(\gamma))+I_{X}(\Theta(\gamma))=N(\gamma)+I_{X}(\gamma)
\end{gathered}
$$

Corollary 2.3. If $\gamma \in \hat{\mathcal{G}_{4}}$ and $k \in \mathbf{Z}$, then

$$
I_{X}\left(\mathcal{T}_{1}^{k}(\gamma)\right)=I_{X}(\gamma) \text { and } N\left(\mathcal{T}_{1}^{k}(\gamma)\right)=N(\gamma)+k I_{X}(\gamma) \text {. }
$$

Now, we are going to construct a homeomorphism of $\overline{\pi \mathcal{I}\left(\mathcal{G}_{4}\right)}$ onto $\hat{\mathbb{R}}$. Following Poénaru's idea [3], we consider the triple $\left(i\left(\gamma, \gamma_{0}\right), i\left(\gamma, \gamma_{\infty}\right), i\left(\gamma, \gamma_{1}\right)\right)$ for $\gamma$ in $\mathcal{G}_{4}$. It is easy to see that $i\left(\gamma, \gamma_{\infty}\right)=2 I_{X}(\gamma)$ and $i\left(\gamma, \gamma_{0}\right)=2|N(\gamma)|$ for $\gamma \in \hat{\mathcal{G}}_{4}$. By Corollary 2.3 , if $\gamma \in \hat{\mathcal{G}}_{4}$, then

$$
i\left(\gamma, \gamma_{1}\right)=i\left(\mathcal{T}_{1}^{-1}(\gamma), \gamma_{0}\right)=2\left|N\left(\mathcal{T}_{1}^{-1}(\gamma)\right)\right|=2\left|N(\gamma)-I_{X}(\gamma)\right| .
$$

For any $\gamma \in \mathcal{G}_{4}$, let $\lambda(\gamma)=i\left(\gamma, \gamma_{0}\right)+i\left(\gamma, \gamma_{\infty}\right)+i\left(\gamma, \gamma_{1}\right)$, and let

$$
x_{1}(\gamma)=\frac{i\left(\gamma, \gamma_{0}\right)}{\lambda(\gamma)}, \quad x_{2}(\gamma)=\frac{i\left(\gamma, \gamma_{\infty}\right)}{\lambda(\gamma)} \quad \text { and } \quad x_{3}(\gamma)=\frac{i\left(\gamma, \gamma_{1}\right)}{\lambda(\gamma)} .
$$

Let $\psi: \mathcal{G}_{4} \longrightarrow \mathbb{R}_{+}^{3}$ be defined by

$$
\psi(\gamma)=\left(x_{1}(\gamma), x_{2}(\gamma), x_{3}(\gamma)\right) .
$$

A direct computation shows that the image of $\psi$ is contained in the triangle $\Delta \subset \mathbb{R}^{3}$ with the vertices $\left(\frac{1}{2}, 0, \frac{1}{2}\right),\left(0, \frac{1}{2}, \frac{1}{2}\right)$ and $\left(\frac{1}{2}, \frac{1}{2}, 0\right)$ (see Figure 3 ). Moreover,

$$
\begin{aligned}
\psi\left(\sigma_{1} \cup \sigma_{2} \cup \sigma_{3}\right) & \subset\left\{\left(x_{1}, x_{2}, x_{3}\right) \in \mathbb{R}_{+}^{3}: x_{1}+x_{2}+x_{3}=1 \text { and } x_{3}=x_{1}+x_{2}\right\}, \\
\psi\left(\sigma_{4}\right) & \subset\left\{\left(x_{1}, x_{2}, x_{3}\right) \in \mathbb{R}_{+}^{3}: x_{1}+x_{2}+x_{3}=1 \text { and } x_{2}=x_{1}+x_{3}\right\}, \\
\psi\left(\sigma_{5} \cup \sigma_{6}\right) & \subset\left\{\left(x_{1}, x_{2}, x_{3}\right) \in \mathbb{R}_{+}^{3}: x_{1}+x_{2}+x_{3}=1 \text { and } x_{1}=x_{2}+x_{3}\right\} .
\end{aligned}
$$

Next, we define $\Psi: \hat{\mathcal{G}}_{4} \longrightarrow \mathbb{R}$ as follows:

$$
\Psi(\gamma)=\left\{\begin{array}{ll}
\frac{x_{1}(\gamma)}{x_{2}(\gamma)} & \text { if } N(\gamma) \geq 0 \\
-\frac{x_{1}(\gamma)}{x_{2}(\gamma)} & \text { if } N(\gamma) \leq 0
\end{array}\right\}=\frac{N(\gamma)}{I_{X}(\gamma)} .
$$


Lemma 2.4. The two functions $\psi$ and $\Psi$ are injective. Moreover, $\Psi \mathcal{T}_{1}(\gamma)=$ $\Psi(\gamma)+1, \Psi \Theta(\gamma)=-\Psi(\gamma)$, and the image of $\Psi$ is $\mathbf{Q}$.

Proof. By Lemma 2.2,

$$
\begin{aligned}
\Psi \mathcal{T}_{1}(\gamma) & =\frac{N\left(\mathcal{T}_{1}(\gamma)\right)}{I_{X}\left(\mathcal{T}_{1}(\gamma)\right)}=\frac{N(\gamma)+I_{X}(\gamma)}{I_{X}(\gamma)}=\Psi(\gamma)+1 \\
\Psi \Theta(\gamma) & =\frac{N(\Theta(\gamma))}{I_{X}(\Theta(\gamma))}=\frac{-N(\gamma)}{I_{X}(\gamma)}=-\Psi(\gamma) .
\end{aligned}
$$

Let $r$ be any non-negative rational number, and let $m \geq 0$ be the integer such that $0 \leq r-m<1$. There exist integers $a>0, b \geq 0$ with $\operatorname{gcd}(a, b)=1$ such that $r-m=\frac{b}{a+b}$. Then

$$
\begin{aligned}
\Psi\left(a \gamma_{0}+b \gamma_{1}\right) & =\frac{b}{a+b}=r-m, \\
\Psi \mathcal{T}_{1}^{m}\left(a \gamma_{0}+b \gamma_{1}\right) & =\Psi\left(a \gamma_{0}+b \gamma_{1}\right)+m=r, \text { and } \\
\Psi \Theta \mathcal{T}_{1}^{m}\left(a \gamma_{0}+b \gamma_{1}\right) & =-\Psi \mathcal{T}_{1}^{m}\left(a \gamma_{0}+b \gamma_{1}\right)=-r .
\end{aligned}
$$

This proves that the image of $\Psi$ is $\mathbf{Q}$.

Next, we shall prove that $\Psi$ is injective. Assume that $\gamma, \gamma^{\prime} \in \hat{\mathcal{G}_{4}}$ satisfy $\Psi(\gamma)=$ $\Psi\left(\gamma^{\prime}\right)$. Since $\gamma=\gamma^{\prime}$ iff $\mathcal{T}_{1}^{k}(\gamma)=\mathcal{T}_{1}^{k}\left(\gamma^{\prime}\right)$ for any fixed integer $k$, then, by Lemma 2.1 , we may assume that $\gamma=a \gamma_{0}+b \gamma_{1}$. Thus, $0 \leq \Psi(\gamma) \leq 1$.

If $\gamma^{\prime}$ is not in $\sigma_{4}$, then, by Lemma 2.1 again, there is an integer $k \neq 0$ such that $\mathcal{T}_{1}^{k}\left(\gamma^{\prime}\right) \in \sigma_{4}$. If

$$
\Psi \mathcal{T}_{1}^{k}\left(\gamma^{\prime}\right)=\Psi\left(\gamma^{\prime}\right)+k \text { and } 0 \leq \Psi \mathcal{T}_{1}^{k}\left(\gamma^{\prime}\right) \leq 1,
$$

then $\Psi\left(\gamma^{\prime}\right)$ is not in the interval $[0,1]$. This is a contradiction since $\Psi(\gamma)=\Psi\left(\gamma^{\prime}\right)$.

Write $\gamma^{\prime}=c \gamma_{0}+d \gamma_{1}$. By assumption, $\frac{d}{c+d}=\frac{b}{a+b}$. Since $\operatorname{gcd}(a, b)=\operatorname{gcd}(c, d)=1$, then $a=c$ and $b=d$, and thus $\gamma=\gamma^{\prime}$. This proves that $\Psi$ is injective.

Now, we prove that $\psi$ is injective. Let $\Delta$ be the triangle as given above, and let $\Delta^{\prime}$ be the image of $\Delta$ under the orthogonal projection

$$
p\left(x_{1}, x_{2}, x_{3}\right)=\left(x_{1}, x_{2}\right) \quad \text { for }\left(x_{1}, x_{2}, x_{3}\right) \in \Delta .
$$

It is easy to see that $p$ is a homeomorphism of $\Delta$ onto $\Delta^{\prime}$. Let $q: \Delta^{\prime}-\left\{\left(\frac{1}{2}, 0\right)\right\} \longrightarrow \mathbb{R}$ be defined by

$$
q\left(x_{1}, x_{2}\right)= \begin{cases}-\frac{x_{1}}{x_{2}} & \text { if } x_{1}+x_{2}=\frac{1}{2}, \\ \frac{x_{1}}{x_{2}} & \text { otherwise. }\end{cases}
$$

Clearly, $q$ is a homeomorphism of $\Delta^{\prime}$ onto $\mathbb{R}$, and $\Psi(\gamma)=q p \psi(\gamma)$ for all $\gamma \in \hat{\mathcal{G}}_{4}$. Then $\psi$ is injective on $\hat{\mathcal{G}}_{4}$, and thus it is injective on $\mathcal{G}_{4}$. The proof of the lemma is complete.

Now, using notations given in the previous section, we shall extend $\psi$ and $\Psi$ to the closure of $\pi \mathcal{I}\left(\mathcal{G}_{4}\right)$ in $\mathrm{P}\left[\mathcal{G}_{4}, \mathbb{R}_{+}\right]$. First, for every $\gamma \in \mathcal{G}_{4}$ and for each $k=1$, 2 , 3, we define $x_{k}\left(\mathrm{I}_{\gamma}\right)=x_{k}(\gamma)$. For any positive number $t$ and for any $\alpha \in \mathcal{G}_{4}$, $t \mathrm{I}_{\gamma}(\alpha)=t i(\gamma, \alpha)$. Since $x_{k}$ is homogeneous, then we can extend $x_{k}$ to $\pi^{-1} \pi \mathcal{I}\left(\mathcal{G}_{4}\right)$ by defining $x_{k}\left(t \mathrm{I}_{\gamma}\right)=x_{k}\left(\mathrm{I}_{\gamma}\right)$ for all $\gamma \in \mathcal{G}_{4}$ and for all positive numbers $t$. Thus, $x_{k}$ induces a well-defined map on $\pi \mathcal{I}\left(\mathcal{G}_{4}\right)$ given by $x_{k}\left(\pi\left(\mathrm{I}_{\gamma}\right)\right)=x_{k}\left(\mathrm{I}_{\gamma}\right)$.

Let $\ell$ be any point of $\pi^{-1} \overline{\pi \mathcal{I}\left(\mathcal{G}_{4}\right)}$. There is a sequence $\left\{t_{n}\right\}_{n=1}^{\infty}$ of positive numbers, and a sequence $\left\{\alpha_{n}\right\}_{n=1}^{\infty}$ in $\mathcal{G}_{4}$ such that $\left\{t_{n} \mathrm{I}_{\alpha_{n}}\right\}_{n=1}^{\infty}$ converges to $\ell$. For 
$m=0,1$ or $\infty, t_{n} \mathrm{I}_{\alpha_{n}}\left(\gamma_{m}\right) \longrightarrow \ell\left(\gamma_{m}\right)$, then

$$
\begin{aligned}
x_{1}\left(t_{n} \mathrm{I}_{\alpha_{n}}\right) & \longrightarrow \frac{\ell\left(\gamma_{0}\right)}{\ell\left(\gamma_{0}\right)+\ell\left(\gamma_{1}\right)+\ell\left(\gamma_{\infty}\right)}, \\
x_{2}\left(t_{n} \mathrm{I}_{\alpha_{n}}\right) & \longrightarrow \frac{\ell\left(\gamma_{\infty}\right)}{\ell\left(\gamma_{0}\right)+\ell\left(\gamma_{1}\right)+\ell\left(\gamma_{\infty}\right)}, \\
x_{3}\left(t_{n} \mathrm{I}_{\alpha_{n}}\right) & \longrightarrow \frac{\ell\left(\gamma_{1}\right)}{\ell\left(\gamma_{0}\right)+\ell\left(\gamma_{1}\right)+\ell\left(\gamma_{\infty}\right)} .
\end{aligned}
$$

Let $\lambda: \pi^{-1} \overline{\pi \mathcal{I}\left(\mathcal{G}_{4}\right)} \longrightarrow \mathbb{R}_{+}$be defined by

$$
\lambda(\ell)=\ell\left(\gamma_{0}\right)+\ell\left(\gamma_{1}\right)+\ell\left(\gamma_{\infty}\right),
$$

and let $x_{k}: \pi^{-1} \overline{\pi \mathcal{I}\left(\mathcal{G}_{4}\right)} \longrightarrow \mathbb{R}_{+}$be defined by

$$
x_{1}(\ell)=\frac{\ell\left(\gamma_{0}\right)}{\lambda(\ell)}, \quad x_{2}(\ell)=\frac{\ell\left(\gamma_{\infty}\right)}{\lambda(\ell)}, \quad x_{3}(\ell)=\frac{\ell\left(\gamma_{1}\right)}{\lambda(\ell)} .
$$

It is easy to see that $x_{k}(t \ell)=x_{k}(\ell)$ for all $t>0$ and for all $\ell \in \pi^{-1} \overline{\pi \mathcal{I}\left(\mathcal{G}_{4}\right)}$. Then $x_{k}$ induces a well-defined map on $\overline{\pi \mathcal{I}\left(\mathcal{G}_{4}\right)}$ defined by

$$
x_{k}(\pi(\ell))=x_{k}(\ell) \quad \text { for all } \ell \in \pi^{-1} \overline{\pi \mathcal{I}\left(\mathcal{G}_{4}\right)} .
$$

It follows immediately from the definition that $x_{k}$ is continuous on $\pi^{-1} \overline{\pi \mathcal{I}\left(\mathcal{G}_{4}\right)}$ for $k=1,2$ or 3 . The restriction of $\pi$ to $\pi^{-1} \overline{\pi \mathcal{I}\left(\mathcal{G}_{4}\right)}$ is a quotient map onto $\overline{\pi \mathcal{I}\left(\mathcal{G}_{4}\right)}$; then $x_{k}$ is continuous on $\overline{\pi \mathcal{I}\left(\mathcal{G}_{4}\right)}$. Now, we obtain a continuous map of $\overline{\pi \mathcal{I}\left(\mathcal{G}_{4}\right)}$ into $\Delta$ whose restriction to $\pi \mathcal{I}\left(\mathcal{G}_{4}\right) \equiv \mathcal{G}_{4}$ agrees with $\psi$, and we obtain a continuous map of $\overline{\pi \mathcal{I}\left(\mathcal{G}_{4}\right)}-\left\{\pi\left(\mathrm{I}_{\gamma_{\infty}}\right)\right\}$ into $\mathbb{R}$ whose restriction to $\pi \mathcal{I}\left(\hat{\mathcal{G}}_{4}\right) \equiv \hat{\mathcal{G}}_{4}$ agrees with $\Psi$. We also denote these two continuous maps by $\psi$ and $\Psi$, respectively.

Let $p: \Delta \longrightarrow \Delta^{\prime}$ and $q: \Delta^{\prime}-\left\{\left(\frac{1}{2}, 0\right)\right\} \longrightarrow \mathbb{R}$ be the two homeomorphisms given in the proof of Lemma 2.4. We define $q\left(\frac{1}{2}, 0\right)=\infty$ and $\Psi\left(\pi\left(\mathrm{I}_{\gamma_{\infty}}\right)\right)=\infty$. Then $q$ is a homeomorphism of $\Delta^{\prime}$ onto $\hat{\mathbb{R}}, \Psi$ is a continuous map of $\overline{\pi \mathcal{I}\left(\mathcal{G}_{4}\right)}$ into $\hat{\mathbb{R}}$, and $\Psi(\mathcal{L})=q p \psi(\mathcal{L})$ for all $\mathcal{L} \in \overline{\pi \mathcal{I}\left(\mathcal{G}_{4}\right)}$. Since $\hat{\mathbb{R}}$ and $\overline{\pi \mathcal{I}\left(\mathcal{G}_{4}\right)}$ are compact, and since $\Psi$ is continuous, then $\Psi$ is a homeomorphism if it is bijective.

Let $r$ be an arbitrary point of $\hat{\mathbb{R}}$. There is a sequence $\left\{r_{n}\right\}_{n=1}^{\infty}$ of points in $\hat{\mathbf{Q}}=\mathbf{Q} \cup\{\infty\}$ converging to $r$. By Lemma 2.4, for each $n$ there is an $\mathcal{L}_{n}$ in $\pi \mathcal{I}\left(\mathcal{G}_{4}\right)$ such that $\Psi\left(\mathcal{L}_{n}\right)=r_{n}$. Since $\overline{\pi \mathcal{I}\left(\mathcal{G}_{4}\right)}$ is compact, then $\left\{\mathcal{L}_{n}\right\}_{n=1}^{\infty}$ has a convergent subsequence, also denoted by $\left\{\mathcal{L}_{n}\right\}_{n=1}^{\infty}$. Let $\mathcal{L} \in \overline{\pi \mathcal{I}\left(\mathcal{G}_{4}\right)}$ be the limit of $\left\{\mathcal{L}_{n}\right\}_{n=1}^{\infty}$. By the continuity of $\Psi$, we have $\Psi(\mathcal{L})=r$. This proves that $\Psi$ is surjective.

In the rest of this section, we shall prove that $\psi$ is injective. This implies that $\Psi$ is injective, and we obtain the following theorem.

Theorem 2.5. The map $\Psi$ is a homeomorphism of $\overline{\pi \mathcal{I}\left(\mathcal{G}_{4}\right)}$ onto $\hat{\mathbb{R}}$. Moreover,

$$
\Psi(\gamma)=\frac{N(\gamma)}{I_{X}(\gamma)} \quad \text { for all } \gamma \in \hat{\mathcal{G}}_{4}, \quad \Psi\left(\gamma_{\infty}\right)=\infty,
$$

and the image of $\mathcal{G}_{4}$ under $\Psi$ is $\hat{\mathbf{Q}}=\mathbf{Q} \cup\{\infty\}$.

To prove the injectivity of $\psi$, we shall need the following theorem.

Theorem 2.6. Let $\gamma$ and $\delta$ be any two distinct elements of $\hat{\mathcal{G}}_{4}$. Then

$$
i(\gamma, \delta)=2\left|I_{X}(\gamma) N(\delta)-I_{X}(\delta) N(\gamma)\right| .
$$


Remark. The equation given in Theorem 2.6 is valid for any two geodesics $\gamma$ and $\delta$ in $\mathcal{G}_{4}$ if we define $I_{X}\left(\gamma_{\infty}\right)=0$ and $N\left(\gamma_{\infty}\right)=1$.

Proof of Theorem 2.6. By Corollary 2.3 we have

$$
I_{X}\left(\mathcal{T}_{1}^{k}(\gamma)\right) N\left(\mathcal{T}_{1}^{k}(\delta)\right)-I_{X}\left(\mathcal{T}_{1}^{k}(\delta)\right) N\left(\mathcal{T}_{1}^{k}(\gamma)\right)=I_{X}(\gamma) N(\delta)-I_{X}(\delta) N(\gamma)
$$

and $i(\gamma, \delta)=i\left(\mathcal{T}_{1}^{k}(\gamma), \mathcal{T}_{1}^{k}(\delta)\right)$ for any integer $k$. Therefore, we may assume $\delta=$ $a \gamma_{2}+b \gamma_{\infty}$ where $a>0$ and $b \geq 0$ are relatively prime integers. Thus, $I_{X}(\delta)=a$ and $N(\delta)=2 a+b$. We shall complete the proof by applying mathematical induction on $I_{X}(\delta)=a$, and we need the following lemma.

Lemma 2.7. Let $\mathcal{T}=\mathcal{T}_{2}^{-1} \Theta \mathcal{T}_{1}^{-1}$. If $\gamma \in \hat{\mathcal{G}}_{4}-\sigma_{6}$, then

$$
I_{X}(\mathcal{T}(\gamma))=2 I_{X}(\gamma)-N(\gamma) \quad \text { and } \quad N(\mathcal{T}(\gamma))=I_{X}(\gamma)-N(\gamma) \text {. }
$$

If $\gamma \in \sigma_{6}-\left\{\gamma_{\infty}\right\}$, then

$$
I_{X}(\mathcal{T}(\gamma))=-2 I_{X}(\gamma)+N(\gamma) \quad \text { and } \quad N(\mathcal{T}(\gamma))=-I_{X}(\gamma)+N(\gamma) .
$$

Proof. By direct computations, we have:

$$
\begin{gathered}
\mathcal{T}_{2}\left(\gamma_{0}\right)=\gamma_{0}, \quad \mathcal{T}_{2}\left(\gamma_{1}\right)=\gamma_{\infty}, \quad \mathcal{T}_{2}\left(\gamma_{0}+\gamma_{1}\right)=\gamma_{1}, \quad \mathcal{T}_{2}\left(\gamma_{0}+2 \gamma_{1}\right)=\gamma_{2}, \\
\mathcal{T}_{2}\left(\gamma_{-1}\right)=\gamma_{0}+\gamma_{-1}, \quad \mathcal{T}_{2}\left(\gamma_{2}\right)=\gamma_{-2}, \quad \mathcal{T}_{2}\left(\gamma_{-2}\right)=\gamma_{0}+2 \gamma_{-1}, \quad \mathcal{T}_{2}\left(\gamma_{\infty}\right)=\gamma_{-1} .
\end{gathered}
$$

Then for any two non-negative relatively prime integers $a$ and $b$ we obtain:

$$
\begin{aligned}
\mathcal{T}\left(a \gamma_{0}+b \gamma_{1}\right) & =(a+b) \gamma_{0}+a \gamma_{1}, \quad \mathcal{T}\left(a \gamma_{0}+b \gamma_{-1}\right)=(a+b) \gamma_{0}+(a+2 b) \gamma_{1}, \\
\mathcal{T}\left(a \gamma_{-1}+b \gamma_{-2}\right) & =(a+b) \gamma_{0}+(2 a+3 b) \gamma_{1}, \quad \mathcal{T}\left(a \gamma_{-2}+b \gamma_{\infty}\right)=a \gamma_{0}+(3 a+b) \gamma_{1} .
\end{aligned}
$$

By the definition of $I_{X}(\gamma)$ and that of $N(\gamma)$, this lemma holds for $\gamma \in \bigcup_{k=1}^{4} \sigma_{k}-$ $\left\{\gamma_{\infty}\right\}$. Next, we consider $a \gamma_{1}+b \gamma_{2}$ :

$$
\begin{aligned}
& \Theta \mathcal{T}_{1}^{-1}\left(a \gamma_{1}+b \gamma_{2}\right)=a \gamma_{0}+b \gamma_{-1}, \\
& a \geq b \Longrightarrow a \gamma_{0}+b \gamma_{-1}=(a-b) \gamma_{0}+b\left(\gamma_{0}+\gamma_{-1}\right) \\
& a<b \leq 2 a \Longrightarrow a \gamma_{0}+b \gamma_{-1}=(2 a-b)\left(\gamma_{0}+\gamma_{-1}\right)+(b-a)\left(\gamma_{0}+2 \gamma_{-1}\right) \\
& \Longrightarrow \mathcal{T}\left(a \gamma_{1}+b \gamma_{2}\right)=(2 a-b) \gamma_{-1}+(b-a) \gamma_{-2}, \\
& b>2 a \Longrightarrow a \gamma_{0}+b \gamma_{-1}=a\left(\gamma_{0}+2 \gamma_{-1}\right)+(b-2 a) \gamma_{-1} \\
& \Longrightarrow \mathcal{T}\left(a \gamma_{1}+b \gamma_{2}\right)=a \gamma_{-2}+(b-2 a) \gamma_{\infty} .
\end{aligned}
$$

The assertions hold for $a \gamma_{1}+b \gamma_{2}$. Finally, we investigate the cases $a \gamma_{2}+b \gamma_{\infty}$, where $a>0$ :

$$
\begin{aligned}
a \geq b & \Longrightarrow a \gamma_{2}+b \gamma_{\infty}=(a-b) \gamma_{2}+b\left(\gamma_{2}+\gamma_{\infty}\right) \\
& \Longrightarrow \mathcal{T}\left(a \gamma_{2}+b \gamma_{\infty}\right)=\mathcal{T}_{2}^{-1} \Theta\left((a-b) \gamma_{1}+b \gamma_{2}\right)=b \gamma_{2}+(a-b) \gamma_{\infty} \\
a<b & \Longrightarrow a \gamma_{2}+b \gamma_{\infty}=a\left(\gamma_{2}+\gamma_{\infty}\right)+(b-a) \gamma_{\infty} \\
& \Longrightarrow \mathcal{T}\left(a \gamma_{2}+b \gamma_{\infty}\right)=\mathcal{T}_{2}^{-1} \Theta\left(a \gamma_{2}+(b-a) \gamma_{\infty}\right)=(b-a) \gamma_{1}+a \gamma_{2}
\end{aligned}
$$

The proof of the lemma is complete.

Now, let $\delta=a \gamma_{2}+b \gamma_{\infty}$ where $a>0$ and $b \geq 0$ are relatively prime integers, and we shall prove that

$$
i(\gamma, \delta)=2\left|I_{X}(\gamma) N(\delta)-I_{X}(\delta) N(\gamma)\right|
$$

for any $\gamma \in \hat{\mathcal{G}}_{4}$ by applying mathematical induction on $I_{X}(\delta)=a$. 
If $a=1$, then $\mathcal{T}_{1}^{-b-2}(\delta)=\gamma_{0}$. Since for any $\gamma \in \hat{\mathcal{G}}_{4}$

$$
i\left(\gamma, \gamma_{0}\right)=2|N(\gamma)|=2\left|I_{X}(\gamma) N\left(\gamma_{0}\right)-I_{X}\left(\gamma_{0}\right) N(\gamma)\right|
$$

then the assertion holds for $\delta=\gamma_{2}+b \gamma_{\infty}$ with $b \geq 0$.

Now, assume that

$$
i\left(\gamma, a^{\prime} \gamma_{2}+b^{\prime} \gamma_{\infty}\right)=2\left|I_{X}(\gamma) N\left(a^{\prime} \gamma_{2}+b^{\prime} \gamma_{\infty}\right)-I_{X}\left(a^{\prime} \gamma_{2}+b^{\prime} \gamma_{\infty}\right) N(\gamma)\right|
$$

for all $\gamma \in \hat{\mathcal{G}}_{4}$ and for all relatively prime integers $a^{\prime}$ and $b^{\prime}$ with $1 \leq a^{\prime}<a$ and $b^{\prime} \geq 0$. Let $\delta$ be given as above. Write $b=k a+c$, where $0<c<a$ and $k \geq 0$. Then $\mathcal{T}_{1}^{-k}(\delta)=a \gamma_{2}+c \gamma_{\infty}$, and by Corollary 2.3 again we may assume $0<b<a$. In this case,

$$
\mathcal{T}(\delta)=b \gamma_{2}+(a-b) \gamma_{\infty}
$$

By the induction hypothesis

$$
i(\gamma, \delta)=i(\mathcal{T}(\gamma), \mathcal{T}(\delta))=2\left|I_{X}(\mathcal{T}(\gamma)) N(\mathcal{T}(\delta))-I_{X}(\mathcal{T}(\delta)) N(\mathcal{T}(\gamma))\right|
$$

By Lemma 2.7, we have

$$
\left|I_{X}(\mathcal{T}(\gamma)) N(\mathcal{T}(\delta))-I_{X}(\mathcal{T}(\delta)) N(\mathcal{T}(\gamma))\right|=\left|I_{X}(\gamma) N(\delta)-I_{X}(\delta) N(\gamma)\right|
$$

This completes the proof of Theorem 2.6.

Proof of the injectivity of $\psi$. It is clear that we only have to show that $\psi$ is injective on $\overline{\pi \mathcal{I}\left(\mathcal{G}_{4}\right)}-\left\{\pi\left(\mathrm{I}_{\gamma_{\infty}}\right)\right\}$.

Let $\mathcal{L}_{1}$ and $\mathcal{L}_{2}$ be any two elements of $\overline{\pi \mathcal{I}\left(\mathcal{G}_{4}\right)}$ with $\psi\left(\mathcal{L}_{1}\right)=\psi\left(\mathcal{L}_{2}\right)$. There exist sequences $\left\{t_{n}\right\}$ and $\left\{s_{n}\right\}$ of positive numbers, and there exist sequences $\left\{\alpha_{n}\right\}$ and $\left\{\beta_{n}\right\}$ in $\hat{\mathcal{G}}_{4}$ such that

$$
\lim _{n \rightarrow \infty} t_{n} \mathrm{I}_{\alpha_{n}}=\ell_{1} \quad \text { and } \quad \lim _{n \rightarrow \infty} s_{n} \mathrm{I}_{\beta_{n}}=\ell_{2},
$$

where $\pi\left(\ell_{j}\right)=\mathcal{L}_{j}$.

By assumption, for $k=0,1$ or $\infty$

$t \ell_{2}\left(\gamma_{k}\right)=\ell_{1}\left(\gamma_{k}\right) \quad$ and $\quad \lim _{n \rightarrow \infty} t_{n} \mathrm{I}_{\alpha_{n}}\left(\gamma_{k}\right)=\lim _{n \rightarrow \infty} t s_{n} \mathrm{I}_{\beta_{n}}\left(\gamma_{k}\right), \quad$ where $\quad t=\frac{\lambda\left(\ell_{1}\right)}{\lambda\left(\ell_{2}\right)}$,

or equivalently

$$
\begin{gathered}
\lim _{n \rightarrow \infty} t_{n} I_{X}\left(\alpha_{n}\right)=\lim _{n \rightarrow \infty} t s_{n} I_{X}\left(\beta_{n}\right), \quad \lim _{n \rightarrow \infty} t_{n}\left|N\left(\alpha_{n}\right)\right|=\lim _{n \rightarrow \infty} t s_{n}\left|N\left(\beta_{n}\right)\right| \text { and } \\
\lim _{n \rightarrow \infty} t_{n}\left|N\left(\alpha_{n}\right)-I_{X}\left(\alpha_{n}\right)\right|=\lim _{n \rightarrow \infty} t s_{n}\left|N\left(\beta_{n}\right)-I_{X}\left(\beta_{n}\right)\right| .
\end{gathered}
$$

Claim. $\lim _{n \rightarrow \infty} t_{n} \mathrm{I}_{\alpha_{n}}(\gamma)=\lim _{n \rightarrow \infty} t s_{n} \mathrm{I}_{\beta_{n}}(\gamma) \quad$ for all $\gamma \in \mathcal{G}_{4}$.

This claim proves that $\ell_{1}=t \ell_{2}$, and $\mathcal{L}_{1}=\mathcal{L}_{2}$. Therefore, $\psi$ is injective.

Proof of the Claim. Let $\psi\left(\mathcal{L}_{1}\right)=\psi\left(\mathcal{L}_{2}\right)=(a, b, c)$.

Case 1. Assume $b=\ell_{1}\left(\gamma_{\infty}\right)=0$. Then

$$
\lim _{n \rightarrow \infty} t_{n} I_{X}\left(\alpha_{n}\right)=\lim _{n \rightarrow \infty} t s_{n} I_{X}\left(\beta_{n}\right)=0 .
$$

Let $\gamma \in \hat{\mathcal{G}}_{4}$ be arbitrary. Obviously

$$
\begin{aligned}
t_{n}\left|N\left(\alpha_{n}\right)\right| I_{X}(\gamma)-t_{n} I_{X}\left(\alpha_{n}\right)|N(\gamma)| & \leq\left|t_{n} I_{X}\left(\alpha_{n}\right) N(\gamma)-t_{n} N\left(\alpha_{n}\right) I_{X}(\gamma)\right| \\
& \leq t_{n}\left|N\left(\alpha_{n}\right)\right| I_{X}(\gamma)+t_{n} I_{X}\left(\alpha_{n}\right)|N(\gamma)|
\end{aligned}
$$


Then, by Theorem 2.6, we have

$$
\lim _{n \rightarrow \infty} t_{n} \mathrm{I}_{\alpha_{n}}(\gamma)=\lim _{n \rightarrow \infty}\left|t_{n} I_{X}\left(\alpha_{n}\right) N(\gamma)-t_{n} N\left(\alpha_{n}\right) I_{X}(\gamma)\right|=\lim _{n \rightarrow \infty} t_{n}\left|N\left(\alpha_{n}\right)\right| I_{X}(\gamma)
$$

By the same reasoning, we have

$$
\lim _{n \rightarrow \infty} t s_{n} \mathrm{I}_{\beta_{n}}(\gamma)=\lim _{n \rightarrow \infty}\left|t s_{n} I_{X}\left(\beta_{n}\right) N(\gamma)-t s_{n} N\left(\beta_{n}\right) I_{X}(\gamma)\right|=\lim _{n \rightarrow \infty} t s_{n}\left|N\left(\beta_{n}\right)\right| I_{X}(\gamma)
$$

This proves the claim for this case.

Case 2. Assume $a=\ell_{1}\left(\gamma_{0}\right)=0$. Then

$$
\lim _{n \rightarrow \infty} t_{n}\left|N\left(\alpha_{n}\right)\right|=\lim _{n \rightarrow \infty} t s_{n}\left|N\left(\beta_{n}\right)\right|=0
$$

and thus

$$
\lim _{n \rightarrow \infty} t_{n} N\left(\alpha_{n}\right)=\lim _{n \rightarrow \infty} t s_{n} N\left(\beta_{n}\right)=0 .
$$

By Theorem 2.6 again,

$$
\lim _{n \rightarrow \infty} t_{n} \mathrm{I}_{\alpha_{n}}(\gamma)=\lim _{n \rightarrow \infty} t s_{n} \mathrm{I}_{\beta_{n}}(\gamma)
$$

Case 3. Assume $a b \neq 0$. By the continuity of $\psi$, one can choose $\alpha_{n}$ and $\beta_{n}$ so that $N\left(\alpha_{n}\right) N\left(\beta_{n}\right)>0$ (see Figure 3 ). Then we have

$$
\lim _{n \rightarrow \infty} t_{n} I_{X}\left(\alpha_{n}\right)=\lim _{n \rightarrow \infty} t s_{n} I_{X}\left(\beta_{n}\right) \quad \text { and } \quad \lim _{n \rightarrow \infty} t_{n} N\left(\alpha_{n}\right)=\lim _{n \rightarrow \infty} t s_{n} N\left(\beta_{n}\right) .
$$

The claim also follows from Theorem 2.6 for this case. The proof of the claim is complete.

\section{Words And a Trace Formula}

In this section, we shall find for each $\gamma \in \mathcal{G}_{4}$ a word $W(\gamma) \in G$ to represent it. Then, we consider the family of quasiconformally conjugates $G_{\mu}$ of $G$. For every $\gamma$, there is a word $W(\gamma ; \mu) \in G_{\mu}$ representing $\gamma$. We shall compute higher order terms of the trace of $W(\gamma ; \mu)$. This section is a part of the author's Ph.D. thesis [2].

First, we restrict our attention to $\gamma \in \sigma_{4}$. Let

$$
W=W(\gamma)=E_{1} E_{2} \cdots E_{k}, \quad E_{j} \in\left\{S^{ \pm}, T^{ \pm}, X^{ \pm}\right\},
$$

be a cyclic reduced word in $G$ representing $\gamma$. Note that $W$ is a loxodromic transformation.

If $X X, X^{-1} X^{-1}, S S$ or $S^{-1} S^{-1}$ is a subword of $W$, then $\gamma$ is not essential or is a curve spiraling around a puncture on $\Sigma_{n}$ (see (1) in Figure 4). This is not allowed. By the same reasoning, $T^{\varepsilon} E^{-\varepsilon} T^{\varepsilon}$ and $E^{\varepsilon} T^{-\varepsilon} E^{\varepsilon}$ cannot be a subword of $W$, whenever $E \in\{S, X\}$ and $\varepsilon= \pm 1$ (see (2) in Figure 4).

If $T^{\varepsilon} E^{\varepsilon} T^{\varepsilon}$ or $E^{\varepsilon} T^{\varepsilon} E^{\varepsilon}$ is a subword of $W, E \in\{S, X\}$ and $\varepsilon= \pm 1$, then $\gamma$ is not simple (see (3) and (4) in Figure 4).

If $E^{-\delta} T^{\varepsilon} E^{\delta}$ is a subword of $W, E \in\{S, X\}$ and $\varepsilon, \delta \in\{ \pm 1\}$, then $\gamma$ is not simple (see (5) and (6) in Figure 4).

If $E^{\delta^{\prime}} T^{p} E^{\delta}$ is a subword of $W, E \in\{S, X\}, \delta^{\prime}, \delta \in\{ \pm 1\}$ and $p \neq 0$ is an integer, then $\gamma$ is not simple.

From the above discussion, we have:

(1) For every fixed $i$, if $j$ is the smallest positive integer such that $E_{i}, E_{i+j} \in$ $\left\{X^{ \pm}\right\}$, then $j \geq 2, E_{i+1}, \ldots, E_{i+j-1} \in\left\{T^{ \pm}, S^{ \pm}\right\}$, and there is a unique $p \in$ $\{i+1, \ldots, i+j-1\}$ such that $E_{p} \in\left\{S^{ \pm}\right\}$. 


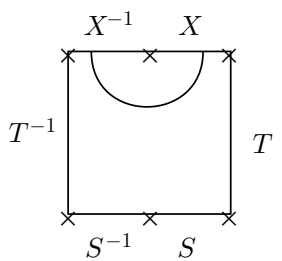

(1)

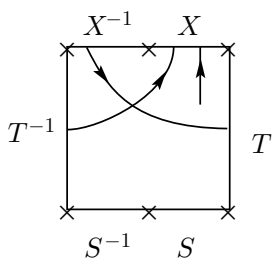

(4)

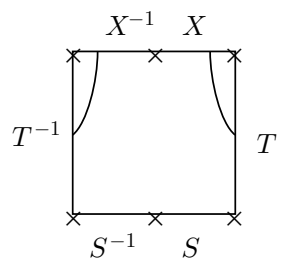

$(2)$

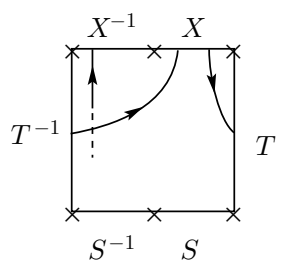

(5)

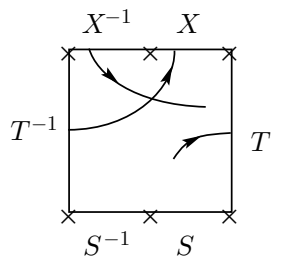

(3)

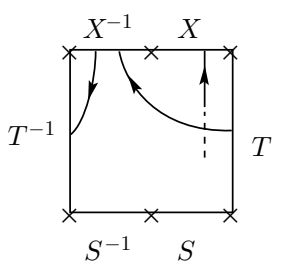

(6)

Figure 4. (1) $X X$ or $X^{-1} X^{-1}$, (2) $T^{-1} X T^{-1}$ or $X^{-1} T X^{-1}$, (3) $T X T$, (4) $X T X$, (5) $X^{-1} T X,(6) X T^{-1} X^{-1}$

(2) For every fixed $i$, if $j$ is the smallest positive integer such that $E_{i}, E_{i+j} \in$ $\left\{S^{ \pm}\right\}$, then $j \geq 2, E_{i+1}, \ldots, E_{i+j-1} \in\left\{T^{ \pm}, X^{ \pm}\right\}$, and there is a unique $p \in$ $\{i+1, \ldots, i+j-1\}$ such that $E_{p} \in\left\{X^{ \pm}\right\}$.

(3) $I_{E}(\gamma)=\#\left\{j: E_{j}=E^{\varepsilon}, 1 \leq j \leq k, \varepsilon= \pm 1\right\}$.

For uniformity, if $X^{\delta} S^{\varepsilon}$ or $S^{\varepsilon} X^{\delta}$ is a subword of $W$, we write

$$
X^{\delta} S^{\varepsilon}=X^{\delta} T^{0} S^{\varepsilon} \text { and } S^{\varepsilon} X^{\delta}=S^{\varepsilon} T^{0} X^{\delta} .
$$

Then $W$ can be written as

$$
\prod_{i=1}^{m} T^{\alpha_{i}} S^{\varepsilon_{i}} T^{\beta_{i}} X^{\delta_{i}}
$$

where $\alpha_{i}, \beta_{i} \in \mathbf{Z}, \varepsilon_{i}, \delta_{i} \in\{1,-1\}$, and $m=I_{X}(\gamma)=I_{S}(\gamma)$. We call a word in $G$ of the form given in equation $(*)$ a cyclic semi-reduced word.

Next, we are going to determine the conditions for $\alpha_{i}, \beta_{i}, \varepsilon_{i}$ and $\delta_{i}$. If $T^{k}$ is a subword of $W$ with $|k| \geq 2$, then $\gamma$ contains a strand joining the $T$-side to the $T^{-1}$ side. Since every curve in $\sigma_{4}$ contains no such strands, then $\left|\alpha_{i}\right| \leq 1$ and $\left|\beta_{i}\right| \leq 1$ for every $i$.

If $T^{-1} S^{\varepsilon} T, \varepsilon= \pm 1$, is a subword of $W$, then $\gamma$ contains a strand joining the $T$-side to the $S$-side, and contains a strand joining the $T$-side to the $S^{-1}$-side, which is impossible for curves in $\sigma_{4}$. Similarly, $T S^{\varepsilon} T^{-1}, T^{-1} X^{\varepsilon} T$ and $T X^{\varepsilon} T^{-1}$ cannot be subwords of $W$. Thus $\alpha_{i} \beta_{i}=0$ and $\alpha_{i+1} \beta_{i}=0$ for $i=1, \ldots, m$, where $\alpha_{m+1}=\alpha_{1}$.

Since $\gamma$ contains no strands joining the $T$-side to the $S^{-1}$-side, and contains no strands joining the $T^{-1}$-side to the $S^{\varepsilon}$-side, $\varepsilon= \pm 1$, then $T^{-1} S^{-1}, S T, T S^{\varepsilon}$ and $S^{\varepsilon} T^{-1}$ cannot be subwords of $W$. Similarly, $X^{-1} T^{-1}, T X, X^{\varepsilon} T$ and $T^{-1} X^{\varepsilon}$ cannot be subwords of $W$. Then

$$
\alpha_{i} \leq 0, \quad \beta_{i} \geq 0, \quad\left(\alpha_{i}+\beta_{i}\right) \varepsilon_{i} \leq 0, \text { and }\left(\alpha_{i+1}+\beta_{i}\right) \delta_{i} \leq 0 .
$$


Proposition 3.1. If $\gamma=a \gamma_{0}+b \gamma_{1}$ with $\operatorname{gcd}(a, b)=1, a, b \geq 0$, and if $W(\gamma) \in G$ is a cyclic semi-reduced word, then $W(\gamma)$ is of the form

$$
\prod_{i=1}^{m} T^{-\alpha_{i}} S^{\varepsilon_{i}} T^{\beta_{i}} X^{\delta_{i}},
$$

where $m=I_{X}(\gamma)=I_{S}(\gamma), \varepsilon_{i}, \delta_{i} \in\{1,-1\}$, and $\alpha_{i}$ and $\beta_{i}$ are non-negative integers satisfying the following conditions:

(1) $\alpha_{i} \leq 1, \beta_{i} \leq 1, \alpha_{i} \beta_{i}=0,\left(\beta_{i}-\alpha_{i}\right) \varepsilon_{i} \leq 0$, for $i=1, \ldots, m$.

(2) $\alpha_{i+1} \beta_{i}=0,\left(\beta_{i}-\alpha_{i+1}\right) \delta_{i} \leq 0$, for $i=1, \ldots, m$, where $\alpha_{m+1}=\alpha_{1}$.

(3) $\sum_{i=1}^{m}\left(\alpha_{i}+\beta_{i}\right)=N(\gamma)$.

Proof. It remains to prove (3). From (1), $\alpha_{i}+\beta_{i} \leq 1$. Let

$$
k=\#\left\{i: \alpha_{i}+\beta_{i}=1\right\} .
$$

Assume that $\alpha_{i}+\beta_{i}=1$. Then

$$
\begin{aligned}
\varepsilon_{i}=-1 & \Longrightarrow \alpha_{i}=0, \beta_{i}=1 \Longrightarrow T^{-\alpha_{i}} S^{\varepsilon_{i}} T^{\beta_{i}}=S^{-1} T, \\
\varepsilon_{i}=1 & \Longrightarrow \alpha_{i}=1, \beta_{i}=0 \Longrightarrow T^{-\alpha_{i}} S^{\varepsilon_{i}} T^{\beta_{i}}=T^{-1} S .
\end{aligned}
$$

Since $S^{-1} T$ or $T^{-1} S$ is a subword of $W$ if and only if $\gamma$ contains a strand joining the $T$-side to the $S$-side, then

$$
\begin{aligned}
k & =\#(\text { strands of } \gamma \text { joining the } T \text {-side to the } S \text {-side }) \\
& =\#\left(\text { strands of } \gamma \text { joining the } T^{-1} \text {-side to the } X^{-1} \text {-side }\right) \\
& =N(\gamma) .
\end{aligned}
$$

Theorem 3.2. If $\gamma \in \hat{\mathcal{G}_{4}}$ and $W(\gamma) \in G$ is a cyclic semi-reduced word representing $\gamma$, then $W(\gamma)$ is of the form

$$
\prod_{i=1}^{m} T^{\alpha_{i}} S^{\varepsilon_{i}} T^{\beta_{i}} X^{\delta_{i}}
$$

where $m=I_{X}(\gamma)=I_{S}(\gamma), \varepsilon_{i}, \delta_{i} \in\{1,-1\}$, and $\alpha_{i}$ and $\beta_{i}$ are integers satisfying the following conditions:

(1) $-1 \leq\left(\alpha_{i}+\beta_{i}\right) \varepsilon_{i} \leq 0$ and $-1 \leq\left(\alpha_{i+1}+\beta_{i}\right) \delta_{i} \leq 0$, where $\alpha_{m+1}=\alpha_{1}$.

(2) $\left|\alpha_{i}\right|,\left|\beta_{i}\right| \in\{\alpha, \alpha+1\}$, where $\alpha=\min \left\{\left|\alpha_{i}\right|,\left|\beta_{i}\right|: i=1, \ldots, m\right\}$.

(3) $\sum_{i=1}^{m}\left(\left|\alpha_{i}\right|+\left|\beta_{i}\right|\right)=|N(\gamma)|$.

(4) $\alpha_{i} \leq 0$ and $\beta_{i} \geq 0$ when $\gamma \in \mathcal{G}_{4}^{+}$, and $\alpha_{i} \geq 0$ and $\beta_{i} \leq 0$ when $\gamma \in \mathcal{G}_{4}^{-}$.

Proof. First, we assume that $\gamma \in \mathcal{G}_{4}^{+}-\sigma_{4}$. By Lemma 2.1, there is an integer $k>0$ such that $\gamma^{\prime}=\mathcal{T}_{1}^{-k}(\gamma) \in \sigma_{4}$. From Corollary 2.3,

$$
I_{X}(\gamma)=I_{X}\left(\gamma^{\prime}\right) \text { and } N(\gamma)=N\left(\gamma^{\prime}\right)+k I_{X}(\gamma) \text {. }
$$

By Proposition 3.1, $\gamma^{\prime}$ is represented by a cyclic semi-reduced word $W^{\prime}$ of the form

$$
\prod_{i=1}^{m} T^{-\alpha_{i}^{\prime}} S^{\varepsilon_{i}^{\prime}} T^{\beta_{i}^{\prime}} X^{\delta_{i}^{\prime}}
$$

where $m=I_{X}(\gamma)=I_{X}\left(\gamma^{\prime}\right), \varepsilon_{i}^{\prime}, \delta_{i}^{\prime} \in\{1,-1\}$, and $\alpha_{i}^{\prime} \geq 0, \beta_{i}^{\prime} \geq 0$ are integers satisfying the conditions given in Proposition 3.1.

Let $\varepsilon^{\prime}, \delta^{\prime} \in\{1,-1\}$ and $\alpha^{\prime} \geq 0, \beta^{\prime} \geq 0$ be integers with $-1 \leq\left(\beta^{\prime}-\alpha^{\prime}\right) \varepsilon^{\prime} \leq 0$. Then

$$
\mathcal{T}_{1}\left(T^{-\alpha^{\prime}} S^{\varepsilon^{\prime}} T^{\beta^{\prime}} X^{\delta^{\prime}}\right)=T^{-\alpha} S^{\varepsilon} T^{\beta} X^{\delta},
$$


where $\varepsilon=\varepsilon^{\prime}, \delta=\delta^{\prime}, \alpha=\alpha^{\prime}+\frac{1}{2}\left(1-\varepsilon^{\prime}\right)$ and $\beta=\beta^{\prime}+\frac{1}{2}\left(1+\varepsilon^{\prime}\right)$. It is easy to see that

$$
\alpha \geq 0, \beta \geq 0, \text { and } \alpha+\beta=\alpha^{\prime}+\beta^{\prime}+1 .
$$

Since

$$
(\beta-\alpha) \varepsilon=-\left(\beta^{\prime}-\alpha^{\prime}\right) \varepsilon^{\prime}-1 \text { and }-1 \leq\left(\beta^{\prime}-\alpha^{\prime}\right) \varepsilon^{\prime} \leq 0,
$$

then $-1 \leq(\beta-\alpha) \varepsilon \leq 0$. Therefore, $W(\gamma)=\mathcal{T}_{1}\left(W^{\prime}\right)$ is of the form

$$
\prod_{i=1}^{m} T^{-\alpha_{i}} S^{\varepsilon_{i}} T^{\beta_{i}} X^{\delta_{i}}
$$

with $\varepsilon_{i}=(-1)^{k} \varepsilon_{i}^{\prime}, \quad \delta_{i}=\delta_{i}^{\prime}, \quad \alpha_{i} \geq 0, \quad \beta_{i} \geq 0$,

$$
\alpha_{i}+\beta_{i}=\alpha_{i}^{\prime}+\beta_{i}^{\prime}+k, \text { and }-1 \leq\left(\beta_{i}-\alpha_{i}\right) \varepsilon_{i} \leq 0 .
$$

From Corollary 2.3,

$$
\sum_{i=1}^{m}\left(\alpha_{i}+\beta_{i}\right)=\sum_{i=1}^{m}\left(\alpha_{i}^{\prime}+\beta_{i}^{\prime}+k\right)=N\left(\gamma^{\prime}\right)+k m=N(\gamma) .
$$

Next, we prove that $-1 \leq\left(\beta_{i}-\alpha_{i+1}\right) \delta_{i} \leq 0$ for every $i$. We write

$$
W(\gamma)=\prod_{i=1}^{m} T^{\beta_{i}} X^{\delta_{i}} T^{-\alpha_{i}} S^{\varepsilon_{i}},
$$

and prove that $-1 \leq\left(\beta_{i}-\alpha_{i}\right) \delta_{i} \leq 0$. Let $\Theta^{\prime}$ be the automorphism of $G$ defined by

$$
S \longrightarrow X, T \longrightarrow T, X \longrightarrow S
$$

$\Theta^{\prime}$ induces a homeomorphism of $\Sigma_{4}$ onto itself, which is also denoted by $\Theta^{\prime}$. It is easy to see that $\Theta^{\prime}\left(\mathcal{G}_{4}^{+}\right)=\mathcal{G}_{4}^{-}$. Then $\Theta \Theta^{\prime}(\gamma) \in \mathcal{G}_{4}^{+}$, and

$$
\Theta \Theta^{\prime}(W(\gamma))=\prod_{i=1}^{m} T^{-\beta_{i}} S^{-\delta_{i}} T^{\alpha_{i}} X^{-\varepsilon_{i}} .
$$

Thus

$$
-1 \leq\left(\alpha_{i}-\beta_{i}\right)\left(-\delta_{i}\right) \leq 0 \text {, or equivalently }-1 \leq\left(\beta_{i}-\alpha_{i}\right) \delta_{i} \leq 0 .
$$

Prove that $\alpha_{i}, \beta_{i} \in\{\alpha, \alpha+1\}$. Without loss of generality, we assume that $\alpha=\min \left\{\alpha_{1}, \beta_{1}\right\}$, and assume that $\varepsilon_{1}=1$. Then $\alpha=\beta_{1}$. Since

$$
\mathcal{T}_{1}^{-2}\left(S^{\varepsilon}\right)=T S^{\varepsilon} T^{-1}, \quad \varepsilon= \pm 1
$$

then

$$
\mathcal{T}_{1}^{-2}\left(T^{-\alpha_{i}} S^{\varepsilon_{i}} T^{\beta_{i}}\right)=T^{-\left(\alpha_{i}-1\right)} S^{\varepsilon_{i}} T^{\left(\beta_{i}-1\right)}
$$

and

$$
\mathcal{T}_{1}^{-2 \alpha}(W(\gamma))=\prod_{i=1}^{m} T^{-\alpha_{i}^{\prime}} S^{\varepsilon_{i}} T^{\beta_{i}^{\prime}} X^{\delta_{i}}
$$

where $\alpha_{i}^{\prime}=\alpha_{i}-\alpha, \beta_{i}^{\prime}=\beta_{i}-\alpha$. Suppose that $\alpha_{i}>\alpha+1$ or $\beta_{i}>\alpha+1$ for some $i>1$. Since $\beta_{1}^{\prime}=0$ and $\varepsilon_{1}=1$, then $\mathcal{T}_{1}^{-2 \alpha}(\gamma)$ contains a strand joining the $S^{-1}$-side to the $X^{\delta_{1}}$-side. On the other hand, $\alpha_{i}^{\prime}>1$ or $\beta_{i}^{\prime}>1$ implies that $\mathcal{T}_{1}^{-2 \alpha}(\gamma)$ contains a strand joining the $T$-side to the $T^{-1}$-side, which is impossible since $\gamma$ is simple.

If $\gamma \in \mathcal{G}_{4}^{-}$, we consider the curve $\Theta(\gamma)$. The proof of the theorem is complete. 
Now, we consider the deformation space of $G$. Let $f$ be a quasiconformal homeomorphism of $\hat{\mathbf{C}}$ onto itself such that $f G f^{-1}$ is again a Kleinian group. If $f$ is normalized so that $f$ fixes 0,1 and $\infty$, then $f G f^{-1}$ is the subgroup of $P S L(2, \mathbf{C})$ generated by

$$
S=\left(\begin{array}{cc}
1 & 0 \\
1 & 1
\end{array}\right), \quad T=\left(\begin{array}{ll}
1 & 4 \\
0 & 1
\end{array}\right), \quad X_{\mu}=f X f^{-1}=\left(\begin{array}{cc}
1+\mu & -\mu^{2} \\
1 & 1-\mu
\end{array}\right),
$$

where $\mu \in \mathbf{C}$ satisfies $|\mu| \geq 1$. For every $\mu \in \mathbf{C}-\{0\}$, let $G_{\mu}$ be the subgroup of $P S L(2, \mathbf{C})$ generated by $S, T$, and $X_{\mu}$ given as above, and let

$$
\mathcal{M}_{4}=\left\{\mu \in \mathbf{C}: \operatorname{Im} \mu>0,|\mu| \geq 1 \text { and } G_{\mu} \text { is a Kleinian group }\right\},
$$

where $\mathcal{M}_{4}$ is the Maskit embedding of the Teichmüller space of $\Sigma_{4}$.

For every $\mu \in \mathcal{M}_{4}$, let $\rho_{\mu}: G \longrightarrow G_{\mu}$ be the isomorphism defined by

$$
\rho_{\mu}(S)=S, \quad \rho_{\mu}(T)=T, \quad \rho_{\mu}(X)=X_{\mu} .
$$

For every $\gamma \in \hat{\mathcal{G}}_{4}$, let $W(\gamma ; \mu)=\rho_{\mu}(W(\gamma))$. We write the trace of $W(\gamma ; \mu)$ as

$$
\operatorname{tr} W(\gamma ; \mu)=a_{0} \mu^{d}+a_{1} \mu^{d-1}+O\left(\mu^{d-2}\right),
$$

where $d$ is the degree of $\operatorname{tr} W(\gamma ; \mu)$ and $O\left(\mu^{d-2}\right)$ is a polynomial in $\mu$ of degree $\leq d-2$.

Lemma 3.3. If $\gamma \in \hat{\mathcal{G}_{4}}$, then $\operatorname{tr} W(\Theta(\gamma) ; \mu)=\operatorname{tr} W(\gamma ;-\mu)$.

Proof. Let

$$
C=\left(\begin{array}{cc}
i & 0 \\
0 & -i
\end{array}\right),
$$

let $\chi: P S L(2, \mathbf{C}) \longrightarrow P S L(2, \mathbf{C})$ be defined by

$$
\chi(A)=C A C^{-1}, \text { for } A \in P S L(2, \mathbf{C}),
$$

and let $\Phi=\chi \Theta$. By a direct computation,

$$
\Phi(S)=S, \quad \Phi(T)=T, \quad \Phi\left(X_{\mu}\right)=X_{-\mu} .
$$

Then

$\operatorname{tr} W(\Theta(\gamma) ; \mu)=\operatorname{tr} \Theta(W(\gamma ; \mu))=\operatorname{tr} \chi \Theta(W(\gamma ; \mu))=\operatorname{tr} \Phi(W(\gamma ; \mu))=\operatorname{tr} W(\gamma ;-\mu)$.

Theorem 3.4 (Trace Formula). If $\gamma \in \hat{\mathcal{G}_{4}}$, then

$$
\operatorname{tr} W(\gamma ; \mu)= \pm\left(\mu^{2 m}+4 N(\gamma) \mu^{2 m-1}\right)+O\left(\mu^{2 m-2}\right),
$$

where $m=I_{X}(\gamma)$.

Proof. If $\varepsilon, \delta \in\{1,-1\}$ and $\alpha$ is an integer, then

$$
S^{\varepsilon}=\left(\begin{array}{ll}
1 & 0 \\
\varepsilon & 1
\end{array}\right), \quad T^{\alpha}=\left(\begin{array}{cc}
1 & 4 \alpha \\
0 & 1
\end{array}\right), \quad X^{\delta}=X_{\mu}^{\delta}=\left(\begin{array}{cc}
1+\delta \mu & -\delta \mu^{2} \\
\delta & 1-\delta \mu
\end{array}\right) .
$$

By Lemma 3.3, we assume that $\gamma \in \mathcal{G}_{4}^{+}$. For $i=1, \ldots, m$, let

$$
\begin{aligned}
& T^{-\alpha_{i}} S^{\varepsilon_{i}} T^{\beta_{i}} X^{\delta_{i}}=\left(\begin{array}{cc}
a_{i}(\mu) & b_{i}(\mu) \\
c_{i}(\mu) & d_{i}(\mu)
\end{array}\right), \text { and } \\
& \prod_{i=1}^{m} T^{-\alpha_{i}} S^{\varepsilon_{i}} T^{\beta_{i}} X^{\delta_{i}}=\left(\begin{array}{cc}
A_{m}(\mu) & B_{m}(\mu) \\
C_{m}(\mu) & D_{m}(\mu)
\end{array}\right),
\end{aligned}
$$


where $\alpha_{i} \geq 0, \beta_{i} \geq 0$ and $\sum_{i=1}^{m}\left(\alpha_{i}+\beta_{i}\right)=N(\gamma)$. A direct computation yields:

$$
\begin{aligned}
a_{i}(\mu) & =\left(1-4 \alpha_{i} \varepsilon_{i}\right) \delta_{i} \mu+\text { constant } \\
b_{i}(\mu) & =-\left(1-4 \alpha_{i} \varepsilon_{i}\right) \delta_{i} \mu^{2}-4 \delta_{i}\left(-\alpha_{i}+\beta_{i}-4 \alpha_{i} \beta_{i} \varepsilon_{i}\right) \mu+\text { constant } \\
c_{i}(\mu) & =\varepsilon_{i} \delta_{i} \mu+\text { constant } \\
d_{i}(\mu) & =-\varepsilon_{i} \delta_{i} \mu^{2}-\left(1+4 \varepsilon_{i} \beta_{i}\right) \delta_{i} \mu+\text { constant. }
\end{aligned}
$$

If $m=1$,

$$
\begin{aligned}
\operatorname{tr} W(\gamma ; \mu) & =-\varepsilon_{1} \delta_{1} \mu^{2}+4 \varepsilon_{1} \delta_{1}\left(-\alpha_{1}-\beta_{1}\right) \mu+\text { constant } \\
& =-\varepsilon_{1} \delta_{1}\left(\mu^{2}+4\left(\alpha_{1}+\beta_{1}\right) \mu\right)+\text { constant } \\
& =-\varepsilon_{1} \delta_{1}\left(\mu^{2}+4 N(\gamma) \mu\right)+\text { constant. }
\end{aligned}
$$

Applying induction on $m$, one can show that if $m \geq 2$, then

$$
\begin{aligned}
A_{m} & =(-1)^{m-1}\left(1-4 \alpha_{1} \varepsilon_{1}\right) K_{m} \mu^{2 m-1}+O\left(\mu^{2 m-2}\right), \\
B_{m} & =(-1)^{m}\left(1-4 \alpha_{1} \varepsilon_{1}\right) K_{m} \mu^{2 m}+O\left(\mu^{2 m-1}\right), \\
C_{m} & =(-1)^{m-1} \varepsilon_{1} K_{m} \mu^{2 m-1}+O\left(\mu^{2 m-2}\right), \\
D_{m} & =(-1)^{m} \varepsilon_{1} K_{m} \mu^{2 m}+(-1)^{m} K_{m}\left\{1+4 \varepsilon_{1}\left(\sum_{i=2}^{m} \alpha_{i}+\sum_{i=1}^{m} \beta_{i}\right)\right\} \mu^{2 m-1}+O\left(\mu^{2 m-2}\right),
\end{aligned}
$$

where

Then

$$
K_{m}=\left(\prod_{i=2}^{m} \varepsilon_{i}\right)\left(\prod_{i=1}^{m} \delta_{i}\right)
$$

$\operatorname{tr} W(\gamma ; \mu)=A_{m}(\mu)+D_{m}(\mu)=(-1)^{m}\left(\prod_{i=1}^{m} \varepsilon_{i} \delta_{i}\right)\left(\mu^{2 m}+4 N(\gamma) \mu^{2 m-1}\right)+O\left(\mu^{2 m-2}\right)$.

If $\gamma \in \mathcal{G}_{4}^{-}$, then $\Theta(\gamma) \in \mathcal{G}_{4}^{+}$, and, by Lemma 2.2 and Lemma 3.3, we have

$$
\begin{aligned}
\operatorname{tr} W(\gamma ; \mu) & =\operatorname{tr} W(\Theta(\gamma) ;-\mu) \\
& = \pm\left((-\mu)^{2 m}+4 N(\Theta(\gamma))(-\mu)^{2 m-1}\right)+O\left(\mu^{2 m-2}\right) \\
& = \pm\left(\mu^{2 m}+4 N(\gamma) \mu^{2 m-1}\right)+O\left(\mu^{2 m-2}\right) .
\end{aligned}
$$

The proof is complete.

\section{REFERENCES}

1. J. Birman and C. Series, Algebraic Linearity for an Automorphism of a Surface Group, J. Pure and Appl Algebra, 52 (1988), 227-275. MR 89j:57006

2. Y. Chiang, Pleating Varieties in the Maskit Embeddings of Teichmüller Spaces of Punctured Spheres, The City University of New York, GSUC, Ph. D. Thesis, 1995.

3. A. Fathi, F. Laudenbach, and V. Poénaru, Travaux de Thurston sur les surfaces, Astérisque, 66-67 (1979).

4. L. Keen and C. Series, Pleating coordinates for the Maskit embedding of the Teichmüller space of punctured tori, Topology, 32 (1993), 719-749. MR 95g:32030

5. L. Keen and C. Series, The Riley Slice of Schottky Space, Proc. London Math. Soc. (3) 69 (1994), 72-90. MR 95j:32033

6. B. Maskit, Kleinian Groups, Springer, New York, 1988. MR 90a:30132

4F No. 16 Chung Yang Rd., Taipei, Taiwan, Republic of China 